CHRONIC OBSTRUCTIVE PULMONARY DISEASE

\title{
Hypoxaemia enhances peripheral muscle oxidative stress in chronic obstructive pulmonary disease
}

\author{
C Koechlin, F Maltais, D Saey, A Michaud, P LeBlanc, M Hayot, C Préfaut
}

Thorax 2005;60:834-841. doi: 10.1136/thx.2004.037531

See end of article for authors' affiliations

Correspondence to:

Correspondence to: de Physiologie des Interactions, Service Central de Physiologie Clinique, Hôpital Arnaud de Villeneuve, 34295

Montpellier cedex 5, France;

christelle_koechlin@

hotmail.com

Received

18 November 2004

Accepted 1 June 2005

Published Online First

17 June 2005

\begin{abstract}
Background: Because oxidative stress affects muscle function, the underlying mechanism to explain exercise induced peripheral muscle oxidative stress in patients with chronic obstructive pulmonary disease (COPD) is clinically relevant. This study investigated whether chronic hypoxaemia in COPD worsens peripheral muscle oxidative stress and whether an abnormal muscle inflammatory process is associated with it.

Methods: Nine chronically hypoxaemic and nine non-hypoxaemic patients performed repeated knee extensions until exhaustion. Biopsy specimens were taken from the vastus lateralis muscle before and 48 hours after exercise. Muscle oxidative stress was evaluated by lipid peroxidation (lipofuscin and thiobarbituric acid reactive substances (TBARs)) and oxidised proteins. Inflammation was evaluated by quantifying muscle neutrophil and tumour necrosis factor (TNF)- $\alpha$ levels.

Results: When both groups were taken together, arterial oxygen pressure was positively correlated with quadriceps endurance time $(n=18, r=0.57 ; p<0.05)$. At rest, quadriceps lipofuscin inclusions were significantly greater in hypoxaemic patients than in non-hypoxaemic patients $(2.9(0.2) \vee 2.0(0.3)$ inclusions/fibre; $p<0.05)$. Exercise induced a greater increase in muscle TBARs and oxidised proteins in hypoxaemic patients than in non-hypoxaemic patients $(40.6(9.1) \% v 10.1(5.8) \%$ and $51.2(11.9) \%$ v 3.7 $(12.2) \%$, respectively, both $p=0.01)$. Neutrophil levels were significantly higher in hypoxaemic patients than in non-hypoxaemic patients $\left(53.1\right.$ (11.6) v $21.5(11.2)$ counts per fibre $\left.\times 10^{-3} ; p<0.05\right)$. Exercise did not alter muscle neutrophil levels in either group. Muscle TNF- $\alpha$ was not detected at baseline or after exercise.

Conclusion: Chronic hypoxaemia was associated with lower quadriceps endurance time and worsened muscle oxidative stress at rest and after exercise. Increased muscle neutrophil levels could be a source of the increased baseline oxidative damage. The involvement of a muscle inflammatory process in the exercise induced oxidative stress of patients with COPD remains to be shown.
\end{abstract}

$\mathrm{E}$ xercise induced oxidative stress has been documented in the pulmonary, blood, and muscle compartments of patients with chronic obstructive pulmonary disease (COPD). ${ }^{1-3}$ Because oxidative stress can affect skeletal muscle contractibility and function, ${ }^{4}$ it has been proposed as a potential mechanism of peripheral muscle dysfunction and impaired exercise tolerance in this disease. ${ }^{5}$ Indeed, oral antioxidant supplementation with $\mathrm{N}$-acetylcysteine improves quadriceps endurance in COPD, ${ }^{6}$ supporting a role for exercise induced oxidative stress in diminishing quadriceps endurance in these individuals.

Oxidative stress is considered to be present when the highly reactive oxygen species (ROS) overwhelm the antioxidant defences. The role of hypoxia in modulating oxidative metabolism and production of reactive oxygen derived metabolites has been investigated in several in vitro and animal studies. ${ }^{78}$ Although these experiments have shown that the source of ROS production in hypoxia is likely to be the mitochondria, particularly the respiratory chain,, ${ }^{8}$ the current understanding of the effects of hypoxia on in vivo human oxidative stress is only fragmentary.

The available studies have focused on long term exposure to hypoxic conditions in healthy subjects. In these individuals, oxygen deficient environmental conditions such as those encountered during high altitude expeditions are associated with increased ROS production and oxidative stress in the systemic circulation at rest and during exercise. ${ }^{10}{ }^{11}$ Little is known regarding the effects of hypoxaemia on skeletal muscle oxidative stress in humans. Two studies have reported cumulative oxidative stress and damage in resting muscle of healthy subjects exposed to an oxygen deficient environment. Martinelli and co-workers ${ }^{12}$ reported a threefold increase in basal levels of lipofuscin, a pigment marker of cumulative oxidative stress, in the vastus lateralis of climbers after a high altitude expedition over $5000 \mathrm{~m}$ for 8 weeks. More recently, Lundby and colleagues ${ }^{13}$ reported an increase in resting muscle oxidative DNA damage in seven healthy subjects after 2 weeks at $4100 \mathrm{~m}$. Chronic hypoxaemia, a common physiopathological consequence of COPD, is associated with poor tolerance of peripheral muscle exercise. ${ }^{14}$ The literature on environmental hypoxia suggests that chronic hypoxaemia in COPD could contribute to peripheral muscle oxidative stress under resting conditions and after exercise. ${ }^{12}{ }^{13}$ The primary objective of this study was therefore to test whether chronic hypoxaemia worsens peripheral muscle oxidative stress in patients with COPD at rest and in response to exercise.

The underlying mechanisms for hypoxia induced oxidative stress have yet to be investigated in humans. In vitro studies suggest that inflammation, via the release of cytokines or neutrophils, is one potential pathway. ${ }^{15}$ In cultured cells, hypoxia resulted in an activation of nuclear factor kappa-B, a

\footnotetext{
Abbreviations: $\mathrm{FEV}_{1}$, forced expiratory volume in 1 second; $\mathrm{FVC}$ forced vital capacity; GPx, glutathione peroxidase; HR, heart rate; MCTSA $_{C T}$, mid thigh muscle cross sectional area; MVC, maximal voluntary contraction; $\mathrm{PaO}_{2}$, arterial oxygen pressure; ROS, reactive oxygen species; $\mathrm{SOD}$, superoxide dismutase; $\mathrm{SpO}_{2}$, oxygen pulse saturation; TBARs, thiobarbituric acid reactive substances; TNF- $\alpha$, tumour necrosis factor $\alpha$
} 
central transcription factor involved in the orchestration of inflammatory cascade events. ${ }^{16}{ }^{17}$ In COPD, reduced lung function is associated with increased blood inflammatory markers. ${ }^{18}$ Furthermore, Takabatake and co-workers showed that arterial oxygen tension $\left(\mathrm{PaO}_{2}\right)$ is inversely correlated with circulating tumour necrosis factor alpha (TNF- $\alpha$ ) and its soluble receptor, ${ }^{19}$ suggesting that hypoxaemia in COPD could be associated with the development of a systemic inflammatory process. These results led to the hypothesis that hypoxaemia could worsen muscle oxidative stress through the induction of an inflammatory process in the peripheral skeletal muscles. The second objective of this study was therefore to investigate whether an abnormal inflammatory process occurs at rest and after exercise in the peripheral muscles of hypoxaemic COPD patients.

To achieve these two objectives, we investigated the effect of chronic hypoxaemia on oxidative stress and inflammatory mediators in the vastus lateralis muscle of COPD patients before and after localised muscle exercise designed to minimise the demand on the cardiorespiratory system. ${ }^{14} 20$

\section{METHODS \\ Subjects}

Eighteen ex-smoking men with stable severe COPD as defined by the GOLD guidelines ${ }^{21}$ volunteered to participate in the study. Patients were categorised into two groups according to their resting $\mathrm{PaO}_{2}$ whose cut off value corresponded to the lower normal limit as predicted by the Mellemgaard reference equation. ${ }^{22}$ One group consisted of non-hypoxaemic patients ( $\mathrm{n}=9, \mathrm{PaO}_{2}>$ lower normal limit) and the other consisted of chronic hypoxaemic patients $\left(\mathrm{n}=9, \mathrm{PaO}_{2}<\right.$ lower normal limit). The exclusion criteria were unstable disease (that is, COPD exacerbation in the preceding 2 months), long term supplemental oxygen, sleep related breathing disorders, and other potentially confounding factors such as neuromuscular disease, chronic heart failure, diabetes mellitus, or alcoholism. The pharmacological treatment for all patients included inhaled $\beta$ agonists and/or anticholinergics, and in four cases inhaled corticosteroids. No patients were on theophylline or oral steroid therapy either at study entry or in the preceding 6 months. Patients were considered as sedentary (scores $<9$ ) according to a physical activity questionnaire adapted for older and retired subjects and used for COPD patients. ${ }^{3}$ All participants had a body mass index lower than 30 and all were questioned on their dietary habits to rule out antioxidant or vitamin supplementation. Informed written consent was obtained from all patients and the research protocol was approved by the local institutional ethic committee.

\section{Study design}

Patients were instructed to abstain from strenuous physical activity for 4 days before and 2 days after being studied. On day 1 the subjects underwent spirometric testing, arterial blood sampling, and computed tomographic (CT) scanning of the thigh, and they had to answer the physical activity questionnaire. Strength was evaluated in each leg and a baseline muscle biopsy was performed on the non-dominant leg. On day 2 the subjects were familiarised with the endurance test procedures by performing five consecutive dynamic knee extensions of the dominant leg. They then performed the localised muscle endurance exercise test. On day 4 (48 hours after localised exercise) a biopsy specimen of the dominant quadriceps was obtained.

\section{Study methods}

\section{Pulmonary function tests and blood gas analysis}

All subjects underwent spirometric testing from which forced expiratory volume in 1 second $\left(\mathrm{FEV}_{1}\right)$ and forced vital capacity (FVC) were obtained and the $\mathrm{FEV}_{\mathrm{l}} / \mathrm{FVC}$ ratio calculated. The results of pulmonary function testing were compared with the normal values of Knudson and coworkers. ${ }^{23}$ Arterial blood was drawn from a radial artery while the subject was breathing room air in the seated position. $\mathrm{PaO}_{2}$ was then analysed with a blood gas analyser (AVL 995, AVL Scientific, Roswell, GA, USA).

Mid thigh muscle cross sectional area measurement A CT scan of the dominant thigh (that is, the stronger one) was performed halfway between the pubic symphysis and the inferior condyle of the femur using a fourth generation Toshiba Scanner 900S (Toshiba Inc, Tokyo, Japan). The mid thigh muscle cross sectional area ( MTCSA $_{\mathrm{CT}}$ ) was obtained as previously described. ${ }^{24}$

\section{Quadriceps strength measurement}

The maximal voluntary strength of the quadriceps was measured in a standardised seated position while the subjects performed dynamic knee extensions against a hydraulic resistance (HF STAR, Hydrafitness Total Power; Henley Health Care, Belton, TX, USA) as previously described. ${ }^{3}$

\section{Quadriceps endurance measurement}

Quadriceps endurance was assessed on an exercise bench according to the technique described by Couillard et al. ${ }^{3}$ The exercises were performed with the subject in a seated position at $90^{\circ}$ knee and hip flexion with arms crossed on the chest, and the position maintained to avoid any changes in respiratory responses. The subjects were asked to perform repeated knee extensions of the dominant leg against weights corresponding to $30 \%$ of their maximal voluntary contraction, at a pace of six movements per minute imposed by an audio signal (metronome) until exhaustion. The dynamic knee extension was performed for 3 seconds, immediately followed by active leg return (eccentric flexion) against resistance for 3 seconds and by rest (for 4 seconds) before the next extension. The test was stopped when the subjects could no longer perform a maximal knee extension or maintain the imposed rhythm despite verbal encouragement. The duration of the test was then recorded as the quadriceps endurance time. The sensations of dyspnoea and muscle fatigue were measured at rest and immediately after exercise on a $10 \mathrm{~cm}$ visual analogue scale. Oxygen pulse saturation $\left(\mathrm{SpO}_{2}\right)$ and heart rate were measured throughout both exercise protocols using a pulse oximeter (Ohmeda Biox II 3740 Pulse Oximeter).

\section{Skeletal muscle analysis \\ Muscle biopsies and tissue preparation of the vastus lateralis}

Percutaneous biopsies of the vastus lateralis muscle (200$300 \mathrm{mg}$ ) of the non-dominant leg at baseline and of the dominant leg 48 hours after exercise were performed $10 \mathrm{~cm}$ above the patella as described by Bergström. ${ }^{25}$ The samples for measurement of oxidative stress markers were immediately frozen in liquid nitrogen and then stored at $-80^{\circ} \mathrm{C}$ until processing. For histochemical analysis, the samples were embedded in OCT compound (Tissue-Tek, Miles Inc, Elkhart, IN, USA) and then stored at $-80^{\circ} \mathrm{C}$ until transverse sections were cut using a cryostat Leica Jung CM 3000 (Wetzlar, Germany) at $-20^{\circ} \mathrm{C}$. Each section was verified by light microscopy to ensure proper fibre orientation. All muscle specimens were coded and analysed without knowledge of the subject's clinical state.

\section{Fibre type determination}

Histochemical fibre typing of the biopsy specimens was carried out as described previously. ${ }^{26}$ Briefly, muscle samples 


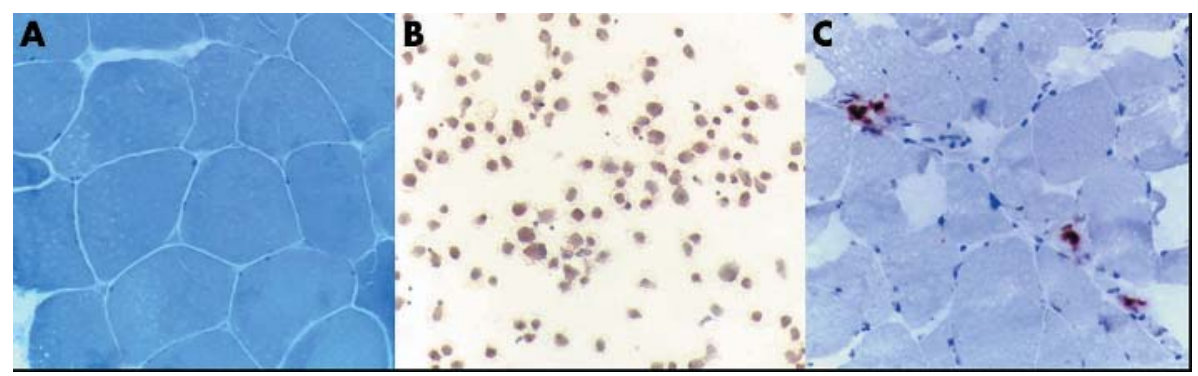

Figure 1 Representative immunohistochemical specificity staining. (A) Lipofuscin inclusions were easily identified as they appeared as dark blue dots in the pale blue green muscle cell cytosol from patients with COPD. Magnification $\times 200$. (B) TNF- $\alpha$ immunopositive staining in bronchoalveolar lavage cells from a patient with asthma. Magnification $\times 20$. (C) Neutrophil immunopositive staining in the muscle cell cytosol from patients with COPD. Magnification $\times 100$.

were cut into $10 \mu \mathrm{m}$ thick transverse sections in a cryostat at $-20^{\circ} \mathrm{C}$ and stained for myofibrillar adenosine triphosphatase activity, according to the single step ethanol modified technique. ${ }^{27}$ Staining intensity designated fibre types as type I (non-stained), type IIa (lightly stained), and type IIx (darkly stained). The mean (SE) total number of fibres counted for muscle fibre typing was 405 (140) (95\% CI 82 to 728 ) and 301 (48) (95\% CI 290 to 412) for the hypoxaemic and non-hypoxaemic groups, respectively.

\section{Determination of muscle oxidative stress markers} Muscle lipofuscin inclusion accumulation, a marker of cumulative oxidative stress, was measured only in preexercise biopsy specimens according to the method previously described by Allaire and co-workers. ${ }^{28}$ Thiobarbituric acid reactive substances (TBARs) were used as markers of muscle lipid peroxidation and were determined spectrophotometrically using the method described by Ohkawa and co-workers. ${ }^{29}$ The final results were expressed in $\mathrm{nmol} / \mathrm{g}$ wet weight. The reproducibility of TBARs, which was calculated as coefficients of variation, was less than $10 \%{ }^{3}$ In the subjects in whom a sufficient amount of muscle tissue was still available (control group, $\mathrm{n}=7$; hypoxaemic group, $n=8$ ), protein oxidation was also measured by evaluating the levels of protein carbonyls using immunoblotting (Oxyblot kit; Serological Corporation, Norcross, GA, USA). Muscle protein carbonyl contents were calculated by adding the integrated density of individual protein bands (Alpha Innotech Corporation, San Leandro, CA, USA) obtained by Western blot analysis. ${ }^{3}$

\section{Determination of muscle antioxidant activity}

The muscle activity of glutathione peroxidase (GPx) and superoxide dismutase (SOD) was quantified spectrophotometrically according to the methods of Nakamura et $a l^{30}$ and Sun et al, ${ }^{31}$ respectively. A unit of SOD activity is defined as the amount of SOD required to cause $50 \%$ inhibition of the oxidation of epinephrine. The reproducibility of these measurements, which were calculated as coefficients of variations, was less than $10 \%$.

Determination of muscle inflammatory mediators The OCT embedded frozen muscle tissue was cut into $10 \mu \mathrm{m}$ sections, mounted on slides, and processed for immunohistochemical analysis. Serial sections were used for immunostaining of TNF- $\alpha$ and neutrophils using human specific antibodies. The tissue sections were fixed at $-20^{\circ} \mathrm{C}$ in acetone/methanol and rinsed in phosphate buffered saline (PBS). To block the non-specific binding of antibodies to tissue, slides were incubated in a humid chamber with a peroxidase blocking reagent $0.3 \%$ for 30 minutes at room temperature (DAKO Diagnostics Canada Inc, Mississauga,
ON, Canada), rinsed with PBS, and then preincubated with a universal blocker (DAKO). Subsequently, sections were incubated at $4^{\circ} \mathrm{C}$ for 2 hours in the humid chamber with monoclonal anti-human TNF- $\alpha$ (Biosource International, Camarillo, CA, USA; dilution $1 / 25$ ) or monoclonal antihuman elastase (DAKO; dilution $1 / 200$ ) primary antibodies. After several washes in PBS, the slides were incubated for 1 hour with horseradish peroxidase secondary antibodies (anti-mouse Ig horseradish peroxidase-link whole antibody, Amersham Biosciences Corporation, Piscataway, NJ, USA; dilution $1 / 100$ in PBS). The sections were then stained using amino ethyl carbazol (AEC) and chromogen as a substrate (DAKO). Slides were counterstained with Mayer's haematoxylin. Positive controls consisted of bronchoalveloar lavage cells from patients with asthma. For negative controls the primary antibody was omitted.

\section{Image analysis and calculations}

Muscle sections were magnified and transmitted to image analysis software (Image Pro Plus 4.1 for Windows; MediaCybernetics, Silver Springs, MD, USA). For each subject the fibre type composition was calculated as the total number of fibres of a given type divided by the total numbers of fibres. The mean fibre size was calculated based on the cross sectional area on 150 randomly selected fibres. ${ }^{26}$ Lipofuscin inclusions were defined as dark blue dots in the pale blue green muscle cell cytosol (fig 1A). Quantification of lipofuscin inclusions was performed at a magnification of $200 \times$ on approximately 150 random fibres. The results are expressed as the lipofuscin/fibre ratio. The muscle inflammatory mediators, as determined by semi-quantitative analyses, are expressed as the total number of positive fibres divided by the total number of fibres (fig 1B, C). All sections were analysed in a blinded fashion and in random order by two observers who were unaware of the clinical data of the case under study. The agreement between the observers was assessed by plotting individual differences between observers' counts against their respective means, as described by Bland and Altman (see fig El in online supplement). ${ }^{32}$

\section{Statistical analysis}

Values are reported as mean (SE) with 95\% confidence intervals (CI). Clinical data and the endurance time were compared between the two groups of COPD patients using an unpaired $t$ test or a Mann-Whitney rank sum test when normality was not obtained. When a $t$ test was performed to compare group mean difference, 95\% CI and p values are noted. When a Mann-Whitney rank sum test based on group median difference was used, only a p value is reported. A two way analysis of variance followed by Tukey's pairwise multiple comparison procedure was used to determine the effects of hypoxaemic status and localised exercise on the 
Table 1 Anthropometric and spirometric data

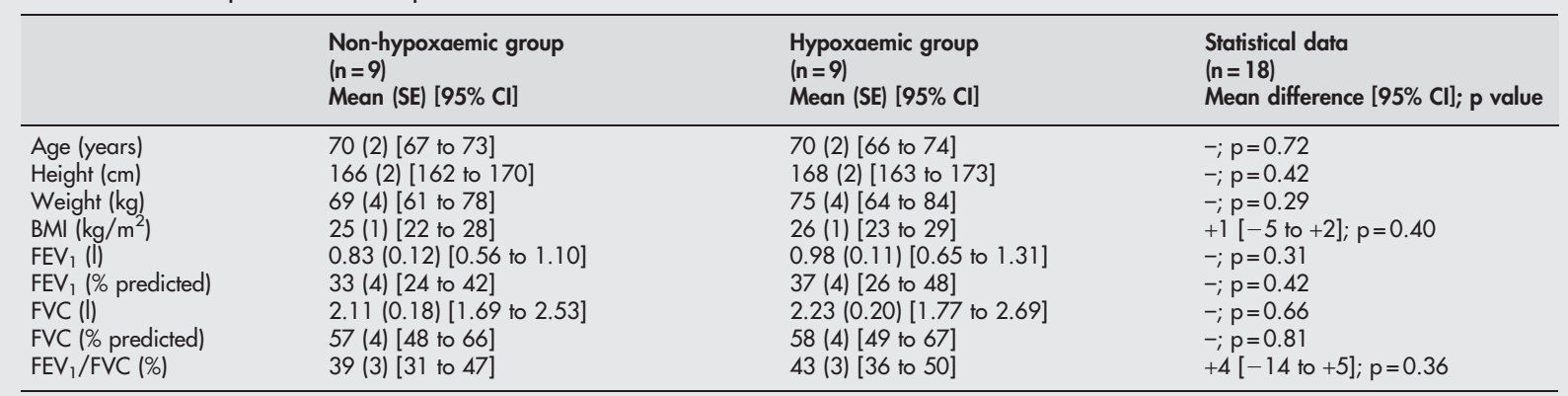

$\mathrm{BMI}$, body mass index; $\mathrm{FEV}_{1}$, forced expiratory volume in 1 second; $\mathrm{FVC}$, forced vital capacity.

When a Mann-Whitney rank sum test based on group median difference was used, only the $p$ value is reported.

biological markers. The significance level was set at 0.05 . The data were analysed using the statistical package Sygmastat 1.0 (Jandel Scientific, San Rafael, CA, USA).

\section{RESULTS}

\section{Anthropometric and spirometric data}

There were no differences in the anthropometric and spirometric data between the two groups (table 1). On average, patients showed severe to very severe airflow obstruction with a mean (SE) $\mathrm{FEV}_{1}$ of 35 (3)\% (95\% CI 29 to 41$)$ of predicted values. ${ }^{23}$

The mean (SE) predicted $\mathrm{PaO}_{2}$ for all patients was 11.34 (0.04) $\mathrm{kPa}$ (95\% CI 11.25 to 11.43 ), with a lower normal value of $9.74(0.04) \mathrm{kPa}$ (95\% CI 9.65 to 9.83). The mean $\mathrm{PaO}_{2}$ averaged $8.69(0.20) \mathrm{kPa}(95 \%$ CI 8.23 to 9.15$)$ and 10.41 (0.26) $\mathrm{kPa}(95 \%$ CI 9.81 to 11.01 ) for the hypoxaemic and non-hypoxaemic groups, respectively (mean difference -1.72 (95\% CI 1.02 to 2.41$) ; p=0.0001$ ). This represented $76(2) \%$ (95\% CI 72 to 80 ) and 92 (2)\% (95\% CI 87 to 97) of the predicted value, respectively (mean difference -15 (95\% CI 10 to 21$) ; \mathrm{p}=0.0001$ ).

\section{Muscle function and local muscle exercise parameters} Mid thigh muscle cross sectional area and maximal voluntary contraction were comparable between the two groups (table 2). Localised muscle endurance tests were performed at a mean load corresponding to $9.0(0.5) \mathrm{kg}$ (95\% CI 7.7 to 10.3). The hypoxaemic group had similar quadriceps endurance time to that of the non-hypoxaemic group (table 2) but, when both groups were taken together, a significant and positive correlation was noted between resting $\mathrm{PaO}_{2}$ and quadriceps endurance time (fig 2). The exercise induced fall in oxygen pulse saturation $\left(\mathrm{SpO}_{2}\right)$ was significantly larger in

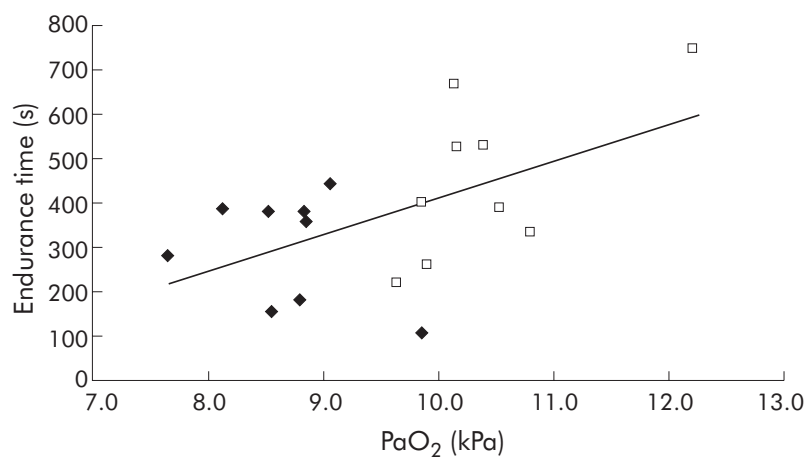

Figure 2 Positive correlation between $\mathrm{PaO}_{2}(\mathrm{kPa})$ and quadriceps endurance time (s) during localised exercise in the 18 patients with COPD (solid symbols represent hypoxaemic patients and open symbols represent non-hypoxaemic patients; $p=0.02, r=0.57$ ). the hypoxaemic group than in the non-hypoxaemic group (table 2). Indeed, $\mathrm{Spo}_{2}$ remained constant throughout exercise in the non-hypoxaemic group while a significant fall in $\mathrm{SpO}_{2}$ was observed in the hypoxaemic group (table 2). The exercise induced increase in heart rate was of small amplitude. The increase in the muscle fatigue score on the visual analogue scale during localised muscle exercise was greater than that of dyspnoea (mean difference -3.1 (95\% CI -4.4 to -1.7$) ; \mathrm{p}=0.0001$ ).

\section{Skeletal muscle data}

Fibre type composition and mean fibre size

The fibre type distribution was characterised by a decrease in type I fibres (32 (4)\% (95\% CI 25 to 39)) and a corresponding increase in type II fibres, with no significant difference between groups (table 3 ). Mean fibre size was also identical between the two groups.

\section{Oxidative stress markers}

More than $90 \%$ of the lipofuscin inclusions observed in our patients were located in the subsarcolemmal area (fig lA). The number of lipofuscin inclusions per fibre in pre-exercise muscle samples was significantly greater in the hypoxaemic group than in the non-hypoxaemic group $(2.9$ (0.2) $v 2.0$ (0.3) lipofuscin inclusions/fibre, respectively; mean difference $+0.9(95 \%$ CI +0.1 to +1.8$) ; p=0.032)$. No significant difference was observed in baseline TBARs (142.4 (119.0) $v$ $155.2(24.2) \mathrm{nmol} / \mathrm{g}$ wet weight; mean difference - 12.8 (95\% CI -70.0 to +44.5$), \mathrm{p}=0.64)$ and protein carbonyl contents (15.3 (4.1) v 16.2 (3.9) integrated density $\times 10^{4}$; mean difference $-0.9(95 \%$ CI -9.6 to +7.8$), p=0.82)$ between the hypoxaemic and non-hypoxaemic groups.

Representative immunoblots of the muscle protein carbonyl group obtained in a hypoxaemic and a non-hypoxaemic patient before and after exercise are shown in fig 3. Four to five bands with molecular weights varying from $20 \mathrm{kDa}$ to $68 \mathrm{kDa}$, including two main 40 and $30 \mathrm{kDa}$ protein bands, were detected in the COPD patients. The baseline migration pattern of these bands was similar between the two groups. After quadriceps exercise these bands became more intense only in the hypoxaemic patients, but no new bands were detected.

The group mean change in muscle TBARs and oxidised proteins 48 hours after exercise are shown in fig 4 for the two groups of patients. There was a greater increase in these oxidative stress markers in the hypoxaemic group than in the non-hypoxaemic group $(\mathrm{p}=0.01)$.

\section{Enzymatic antioxidant activity}

Similar baseline muscle GPx and SOD activities were observed in the two COPD groups (table 4). In both groups, 
Table 2 Muscle function and local muscle exercise parameters

\begin{tabular}{|c|c|c|c|}
\hline & $\begin{array}{l}\text { Non-hypoxaemic group } \\
(\mathrm{n}=9) \\
\text { Mean (SE) }[95 \% \mathrm{Cl}]\end{array}$ & $\begin{array}{l}\text { Hypoxaemic group } \\
(\mathrm{n}=9) \\
\text { Mean (SE) }[95 \% \mathrm{Cl}]\end{array}$ & $\begin{array}{l}\text { Statistical data } \\
(n=18) \\
\text { Mean difference }[95 \% \mathrm{Cl}] \text {, p value }\end{array}$ \\
\hline$M_{C T S A}\left(\mathrm{~cm}^{2}\right)$ & 87 (9) [65 to 109] & 83 (7) [67 to 99] & $+4[-21$ to +29$] ; p=0.74$ \\
\hline$M V C(\mathrm{~kg})$ & 31 (4) [39 to 23] & 30 (2) [25 to 35$]$ & $+1[-8$ to +10$] ; p=0.85$ \\
\hline Quadriceps endurance time (s) & $421(72)$ [255 to 587$]$ & 330 (34) [252 to 408$]$ & $+91[-78$ to +259$] ; p=0.27$ \\
\hline Physical activity score & 4.5 (1.0) $[2.2$ to 6.8$]$ & $3.2(0.5)[2.2$ to 4.2$]$ & $+1.2[-1.1$ to +3.6$] ; p=0.29$ \\
\hline$\Delta \mathrm{HR}$ (pulse/min) & 7 (1) [4 to 10$]$ & 9 (1) [6 to 12$]$ & $-;-; p=0.22$ \\
\hline$\Delta \mathrm{SpO}_{2}(\%)$ & $0.5(0.4)[-0.4$ to +1.4$]$ & $-2.5(0.3)[-3.2 \text { to }+1.0]^{*}$ & $+3.1[+2.1$ to +4.1$] ; \mathbf{p}<\mathbf{0 . 0 0 0 1}$ \\
\hline$\Delta$ Dyspnoea score & $2.9(0.7)[1.3$ to 4.5$]$ & $3.5(0.5)[2.3$ to 4.7$]$ & $+0.5[-2.4$ to +1.3$] ; p=0.53$ \\
\hline$\Delta$ Muscle fatigue score & $6.9(0.6)[5.5$ to 8.3$]$ & $5.7(0.8)[3.9$ to 7.5$]$ & $+1.2[-0.9$ to +3.2$] ; p=0.25$ \\
\hline
\end{tabular}

$\mathrm{MCTSA}_{\mathrm{CT}}$, mid thigh muscle cross sectional area; $\mathrm{MVC}$, maximal voluntary contraction; $\mathrm{HR}$, heart rate; $\mathrm{SpO}_{2}$, oxygen pulse saturation; $\Delta$, change in the corresponding variable from rest to end of exercise.

${ }^{*} \mathrm{p}<0.05$ hypoxaemic $(n=8) \vee$ non-hypoxaemic group $(n=8)$.

exercise failed to induce any increase in GPx or SOD activities.

\section{Inflammatory markers}

The number of neutrophil counts per muscle fibre was higher in the hypoxaemic group than in the non-hypoxaemic group (group effect: 53.1 (11.6) $v 21.5$ (11.2) counts per fibre $\left.\times 10^{-3} ; \mathrm{p}=0.04\right)$. Exercise did not alter the muscle neutrophil levels in either group (table 4). Muscle TNF- $\alpha$ was not detected by immunostaining in the biopsy specimens taken at rest or after exercise in either group of patients.

\section{DISCUSSION}

The major findings of this study are that hypoxaemia worsened muscle oxidative stress in COPD at rest and after localised muscle exercise. Increased muscle neutrophil levels were observed at rest in the muscle biopsy specimens from hypoxaemic patients, suggesting a greater level of muscle inflammation in these individuals. TNF- $\alpha$ was not detected in the biopsy specimens taken either at rest or after exercise.

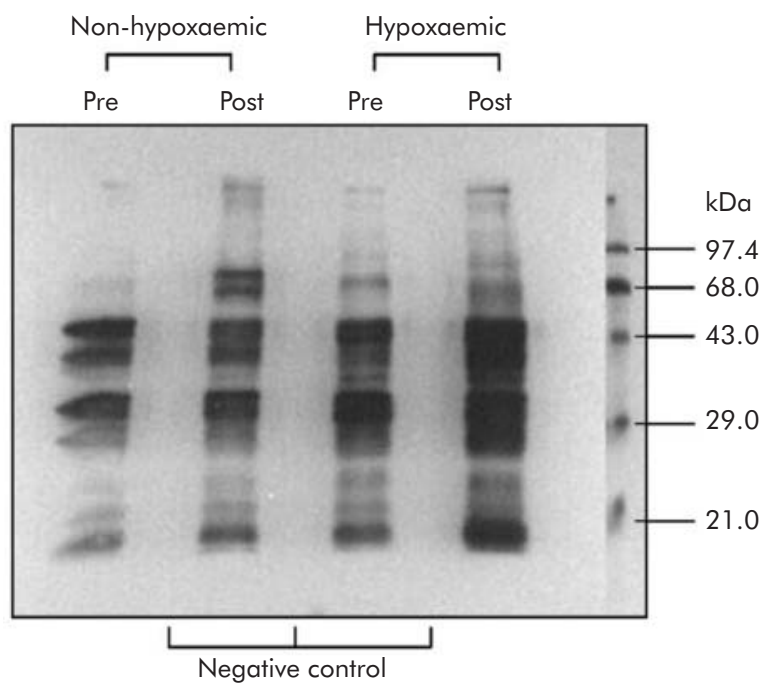

Figure 3 Representative immunoblots of muscle protein carbonyl groups in a non-hypoxaemic patient and a hypoxaemic patient before (pre) and after (post) quadriceps exercise. Four to five bands whose molecular weight varied from 20 to $68 \mathrm{kDa}$ were detected in both patients. Compared with the pre-exercise levels, these bands became more intense in the hypoxaemic patient but no new bands were detected. Negative controls (underivatised protein) are also shown.

\section{Chronic hypoxaemia and peripheral muscle oxidative stress in COPD patients}

Evidence of lipofuscin accumulation, and thus cumulative lipid peroxidation, has been reported in the resting skeletal muscle of patients with COPD. ${ }^{28}$ This study adds to the current knowledge by showing that COPD patients with decreased resting $\mathrm{PaO}_{2}$ have a greater lipofuscin accumulation in the vastus lateralis muscle than patients with normal $\mathrm{PaO}_{2}$. This observation could not be explained by differences in muscle fibre size or composition between the nonhypoxaemic and hypoxaemic patients. The greater muscle accumulation of lipofuscin observed in the hypoxaemic group thus supports the hypothesis that hypoxaemia, as a chronic condition, worsens basal cumulative oxidative damage in COPD skeletal muscle. After exercise the significant increase in muscle TBARs and protein carbonyls (two markers of acute oxidative stress) indicated quadriceps exercise induced oxidative stress, ${ }^{3}$ particularly in the hypoxaemic patients whose muscle TBAR and protein carbonyl levels after exercise were greater than in the non-hypoxaemic patients. These results are therefore consistent with the hypothesis that chronic hypoxaemia worsens peripheral muscle oxidative stress in COPD, both at rest and in response to exercise. The exact mechanisms by which chronic hypoxaemia worsens in vivo peripheral muscle oxidative stress at rest and in response

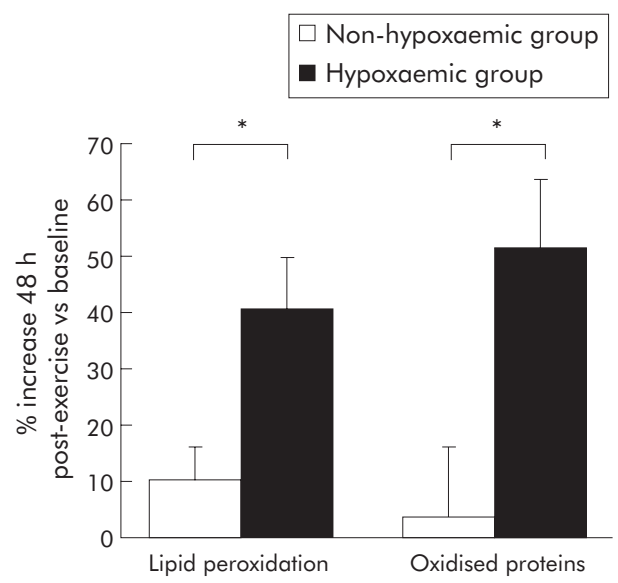

Figure 4 Effects of localised muscle exercise on the increase in muscle lipid peroxidation and oxidised protein levels in the non-hypoxaemic group (open bars; $n=9$ ) and the hypoxaemic group (black bars; $n=9$ ). Both markers of oxidative stress increased markedly after exercise, with a significantly greater increase in the hypoxaemic patients than in the non-hypoxaemic patient $(40.6$ (9.1)\% v 10.1 (5.8)\%; mean difference $+30.4(95 \% \mathrm{Cl}+7.6$ to +53.3$), \mathrm{p}=0.01$ for TBARs; $51.2(11.9) \%$ v 3.7 $(12.2) \%$; mean difference $+47.5(+10.6$ to +84.4$) ; p=0.01$ for oxidised protein). ${ }^{*} p<0.05$ hypoxaemic $v$ non-hypoxaemic group. 
Table 3 Fibre type distribution and fibre size in the non-hypoxaemic and hypoxaemic groups

\begin{tabular}{|c|c|c|c|}
\hline & $\begin{array}{l}\text { Non-hypoxaemic group } \\
(\mathrm{n}=9) \\
\text { Mean (SE) }[95 \% \mathrm{Cl}]\end{array}$ & $\begin{array}{l}\text { Hypoxaemic group } \\
(\mathrm{n}=9) \\
\text { Mean (SE) }[95 \% \mathrm{Cl}]\end{array}$ & $\begin{array}{l}\text { Statistical data } \\
\text { ( } n=18 \text { ) } \\
\text { ANOVA p value }\end{array}$ \\
\hline \multicolumn{4}{|c|}{ Fibre composition (\%) } \\
\hline Type I & 32 (4) [23 to 41$]$ & 31 (7) [16 to 46$]$ & Group effect: $p=0.99$ \\
\hline Type lla & 39 (4) [30 to 48$]$ & 38 (4) [28 to 48$]$ & Type effect: $p=0.12$ \\
\hline Type $l l b$ & 29 (2) [25 to 33$]$ & 30 (5) [19 to 41$]$ & (Group $\times$ fibre type) effect: $p=0.96$ \\
\hline \multicolumn{4}{|l|}{ Fibre size $\left(\mu \mathrm{m}^{2}\right)$} \\
\hline Type I & 5395 (1116) [2527 to 8263$]$ & 5491 (1016) [3043 to 7939] & \\
\hline Type lla & 4839 (1111) [1983 to 7695$]$ & 5157 (1019) [2806 to 7508$]$ & Group effect: $p=0.93$ \\
\hline Type llb & 4056 (1158) [1080 to 7032$]$ & $3838(720)[2178$ to 5498$]$ & Type effect: $p=0.54$ \\
\hline All fibre types & 4764 (626) [3443 to 6085$]$ & 4829 (543) [3712 to 5945$]$ & (Group $\times$ fibre type) effect: $p=0.99$ \\
\hline
\end{tabular}

to exercise remain unknown. Nevertheless, some hypotheses in line with our results can be proposed in COPD.

\section{Potential underlying mechanisms}

Similar changes in the activities of SOD and GPx were observed in the two groups, suggesting that differences in these enzymatic antioxidant defences did not account for the greater muscle oxidative stress observed in hypoxaemic patients. However, the increased neutrophil count observed in the quadriceps of hypoxaemic patients may have contributed to the increased basal oxidative stress in these patients. Indeed, circulating neutrophils are important effector cells in the generation of free radicals via the reduced nicotinamide adenine dinucleotide phosphate (NADPH) oxidase system..$^{33}$ Neutrophils and phagocytes may thus be critical cells in the pathogenesis of hypoxaemia related oxidative stress, supporting the notion that inflammatory mediators are involved in the basal cumulative oxidative stress in the thigh muscle of hypoxaemic COPD patients.

However, no TNF- $\alpha$ could be detected in the resting or post-exercise muscle biopsy specimens of either group of patients. Similarly, no changes in muscle neutrophil levels were observed after exercise. These results may indicate that exercise, which was localised to one muscle group for only a few minutes, was not sufficient to induce an inflammatory process in the muscle of hypoxaemic and non-hypoxaemic COPD patients, despite evidence of exercise induced muscle oxidative stress. However, a role for a muscle inflammatory process in the exercise induced oxidative stress cannot be excluded from the present results. Indeed, a number of mediators other than neutrophils and TNF- $\alpha$ may be involved in the development of an inflammatory process within the muscle. For instance, an increase in nuclear factor NF- $\kappa B$, a key inflammatory transcription factor, ${ }^{35}$ has recently been observed in the skeletal muscles of COPD patients with low body weight. ${ }^{36}$ Furthermore, a possible muscle inflammatory process could have been missed since the post-exercise muscle biopsy specimen was obtained at a single time point. Whether muscle inflammation was present in exercising muscle-and whether it played a role in the muscle oxidative stress-thus remains unclear and needs to be examined further.

Based on the location of lipofuscin pigments, which were mostly in the mitochondria-rich subsarcolemmal area, one possibility is that the mitochondrial electron transportation chain was responsible for the increased ROS production in the skeletal muscle of COPD patients with hypoxaemia. Evidence for increased mitochondrial ROS production has been observed during cellular hypoxia. ${ }^{89}$ The xanthine oxidase system in skeletal muscle is another important source of ROS production in conditions such as the ischaemia-reperfusion phenomenon which is associated with excessive production of free radicals and oxidative injuries. ${ }^{37}$ Based on the increase in blood flow in working muscles during exercise leading to muscle reoxygenation, it may be that exercise in hypoxaemic patients could mimic this ischaemia-reperfusion phenomenon. Given the role of the xanthine oxidase system in inducing oxidative stress during exercise in non-hypoxaemic patients with $\mathrm{COPD}^{38}$ this system is also likely to contribute to the enhanced exercise induced oxidative stress in hypoxaemic patients with COPD.

\section{Hypoxaemia induced muscle oxidative stress and peripheral muscle function}

Evidence of increased molecular oxidative damage, reflected by increased lipid peroxidation and oxidised proteins, was found in the quadriceps of hypoxaemic patients at rest and in response to exercise. However, we did not document functional muscle changes as the strength and endurance of the quadriceps of the hypoxaemic and non-hypoxaemic

Table 4 Muscle enzymatic antioxidant activity and neutrophil counts at rest and after exercise in the non-hypoxaemic and hypoxaemic groups

\begin{tabular}{|c|c|c|c|}
\hline & $\begin{array}{l}\text { Non-hypoxaemic group } \\
(\mathrm{n}=9) \\
\text { Mean (SE) }[95 \% \mathrm{Cl}]\end{array}$ & $\begin{array}{l}\text { Hypoxaemic group } \\
(n=9) \\
\text { Mean (SE) }[95 \% \mathrm{Cl}]\end{array}$ & $\begin{array}{l}\text { Statistical data } \\
\text { ( } n=18) \\
\text { ANOVA p value }\end{array}$ \\
\hline $\begin{array}{l}\text { GPx activity }(\mu \mathrm{mol} / \mathrm{g} / \mathrm{min}) \\
\text { Baseline } \\
\text { Post-exercise }\end{array}$ & $\begin{array}{l}4.0(0.3)[3.2 \text { to } 4.8] \\
3.5(0.2)[3.1 \text { to } 3.9]\end{array}$ & $\begin{array}{l}4.0(0.3)[3.3 \text { to } 4.7] \\
3.8(0.3)[3.1 \text { to } 4.5]\end{array}$ & $\begin{array}{l}\text { Group effect: } p=0.77 \\
\text { Exercise effect: } p=0.23 \\
\text { (Group } \times \text { exercise) effect: } p=0.60\end{array}$ \\
\hline $\begin{array}{l}\text { SOD activity (units) } \\
\text { Baseline } \\
\text { Post-exercise }\end{array}$ & $\begin{array}{l}732.5(34.2)[653.6 \text { to } 811.4] \\
775.1(8.4)[755.8 \text { to } 794.4]\end{array}$ & $\begin{array}{l}737.5(34.2)[634.5 \text { to } 831.5] \\
755.0(7.5)[737.7 \text { to } 772.3]\end{array}$ & $\begin{array}{l}\text { Group effect: } p=0.78 \\
\text { Exercise effect: } p=0.28 \\
\text { (Group } \times \text { exercise) effect: } p=0.65\end{array}$ \\
\hline $\begin{array}{l}\text { Neutrophil counts (per fibre } \times 10^{-3} \text { ) } \\
\text { Baseline } \\
\text { Post-exercise }\end{array}$ & $\begin{array}{l}23.8(7.0)[7.6 \text { to } 40] \\
19.3(3.3)[11.7 \text { to } 26.9]\end{array}$ & $\begin{array}{l}54.3(29.1)[-12.8 \text { to }+83.4]^{*} \\
51.9(9.4)[29.7 \text { to } 74.1]^{*}\end{array}$ & $\begin{array}{l}\text { Group effect: } p=0.04 \\
\text { Exercise effect: } p=0.39 \\
\text { (Group } \times \text { exercise) effect: } p=0.54\end{array}$ \\
\hline
\end{tabular}


patients were not statistically different. This may suggest that the molecular oxidative damage associated with the mild hypoxaemia in this study was not sufficient to alter muscle function. Findings from studies carried out at high altitude suggest that peripheral muscle strength is not affected by oxygen deficient environmental conditions. ${ }^{39} 40$ Although acute or chronic exposure to hypobaric hypoxia in healthy subjects impairs large muscle exercise performance, the effects of hypoxic conditions on the endurance of isolated small muscle groups are poorly defined.$^{41}$ Nevertheless, some studies have shown reduced local muscle endurance under hypoxic conditions in healthy subjects and in patients with COPD ${ }^{41}{ }^{42}$ In the present study we found that quadriceps endurance in COPD patients was inversely correlated with the severity of hypoxaemia, as previously shown by Serres et al. ${ }^{13}$ Because higher muscle oxidative stress was observed in the hypoxaemic patients in our study, and oxidative stress has recently been shown to alter quadriceps muscle endurance in patients with COPD, ${ }^{6}$ we can hypothesise that peripheral muscle oxidative stress may be a deleterious in vivo mechanism through which hypoxaemia could lead to skeletal muscle dysfunction in COPD. ${ }^{2}$

\section{Methodological considerations \\ Hypoxaemic status and analysis of patient subgroups}

We specifically excluded from the study patients qualifying for long term oxygen therapy $\left(\mathrm{PaO}_{2}<7.3-8.0 \mathrm{kPa}\right)$. In these patients it would be unethical to delay oxygen therapy for research purposes and we wished to avoid the potential confounding effects of supplemental oxygen on oxidative stress. We used the Mellemgaard reference equation usually used in clinical hospital practice to detect hypoxaemia, since it takes into account the age in determining the cut off value of predicted normal $\mathrm{PaO}_{2}$ values. ${ }^{22}$ The hypoxaemic group

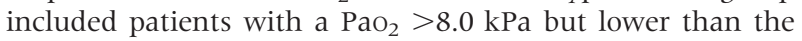
lower normal limit based on the Mellemgaard predictive equation..$^{22}$ Despite being only mildly hypoxaemic, their oxygenation status was clearly different from that of the non-hypoxaemic group (mean resting $\mathrm{PaO}_{2} 1.72$ (0.41) kPa lower than in the non-hypoxaemic group and, in contrast to the latter group, they showed a significant fall in $\mathrm{Spo}_{2}$ during exercise). Given that our two subgroups of patients were otherwise comparable, it is reasonable to assume that differences in blood oxygenation were involved in the observed difference in the peripheral oxidative stress and inflammatory status between them.

\section{Quadriceps exercise protocol}

The localised muscle exercise used in the study to assess muscle endurance has been discussed previously in terms of its reproducibility and specificity to the quadriceps. ${ }^{3}$ Previous electromyographic recordings of the thigh indicated that only the quadriceps was active during this localised exercise. ${ }^{20}$ The increases in dyspnoea sensation and heart rate following exercise were of small amplitude. In contrast, the increase in the perception of muscle fatigue was higher, suggesting that the main reason for stopping exercise was peripheral and not related to respiratory or cardiovascular limitations.

\section{Markers of muscle oxidative stress}

A useful feature of lipofuscin is its tendency to accumulate slowly over time, as it cannot be eliminated effectively. ${ }^{43}$ Since lipofuscin accumulation reflects the magnitude of lipid peroxidation, it can thus be used as a marker of cumulative oxidative stress and has therefore been evaluated only in preexercise biopsy specimens. ${ }^{28}{ }^{43}$ In contrast, TBARs were used as a marker of oxidant mediated lipid damage induced by exercising muscle. One potential limitation of TBARs is that, under oxidative stress conditions, malondialdehyde, hydroperoxides, and some carbohydrates and amino acids may yield products that are able to react with thiobarbituric acid. ${ }^{44}$ However, the spectrophotometric method that we used was sufficiently sensitive and reproducible to provide a valid estimation of oxidative stress, ${ }^{29}$ as previously discussed. ${ }^{3}$ Protein carbonyls are sensitive indices of oxidative injury ${ }^{45}$ and the detection of muscle oxidised proteins provided another fingerprint of exercise oxidative damage and strong evidence of exercise induced oxidative stress in our patients.

\section{Timing of the post-exercise biopsy}

The timing of peak values for an exercise induced increase in muscle oxidative stress markers is a highly debatable issue and, according to previous studies, has been found to vary from immediately after exercise to 72 hours after exercise. ${ }^{46-49}$ In this study the biopsy specimens were obtained 48 hours after exercise, as in a previous study by our group which showed increased oxidative stress in the quadriceps of nonhypoxaemic COPD patients 48 hours after an exercise protocol similar to the one used here. ${ }^{3}$ Since the mitochondria respiratory chain is one of the major sources of oxidant production during exercise, one can predict that the highest rate of ROS production and increased muscle lipid peroxidation should occur immediately after the cessation of exercise. However, ROS were not directly measured; rather, indirect markers of their presence (TBARs and oxidised proteins) which could be detected for a much longer period of time were evaluated. ${ }^{46-49}$ The timing of the second biopsy thus appears appropriate in evaluating the exercise induced muscle inflammation response. ${ }^{50}$

\section{Conclusion}

This study shows that chronic hypoxaemia in COPD patients worsened peripheral muscle oxidative stress both at rest and during exercise. An increase in muscle neutrophil levels could be one potential mechanism of oxidative stress in skeletal muscle at rest. The presence and role of a muscle inflammatory process in the worsening of oxidative stress after exercise remain to be demonstrated. Understanding the underlying mechanisms by which hypoxaemia affects peripheral muscle oxidative stress in COPD will probably lead to the development of better therapeutic approaches for peripheral muscle dysfunction in this disease.

\section{ACKNOWLEDGEMENTS}

The authors thank Marie-Josée Breton, Marthe Bélanger, and Catherine Stott-Carmeni for valuable technical assistance.

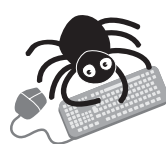

Figure $\mathrm{El}$ is shown in the online supplement available at http://www.thoraxinl.com/supplemental

\footnotetext{
Authors' affiliations

C Koechlin, M Hayot, C Préfaut, UPRES-EA 701, Laboratory of Physiologie des Interactions, Service Central de Physiologie Clinique, Hôpital Arnaud de Villeneuve, 34295 Montpellier cedex 5, France F Maltais, D Saey, A Michaud, P LeBlanc, Centre de Recherche, Hôpital Laval, Institut Universitaire de Cardiologie et de Pneumologie de I'Université Laval, Sainte-Foy, Québec, Canada

This study was supported in part by a Canadian Institutes of Health Research grant number MOP-53135

Competing interests: CK was supported by a travelling grant from La Cooperation Franco-Québécoise. FM is a research scholar of the Fonds de la Recherche en Santé du Québec. DS is a recipient of a PhD training
} award from the Fonds de la Recherche en Santé du Québec. 


\section{REFERENCES}

1 Rahman I, MacNee W. Oxidant/antioxidant imbalance in smokers and chronic obstructive pulmonary disease. Thorax 1996;51:348-50.

2 Langen RCJ, Korn SH, Wouters EFM. ROS in the local and systemic pathogenesis of COPD. Free Radic Biol Med 2003;35:226-35.

3 Couillard A, Maltais F, Saey D, et al. Exercise-induced quadriceps oxidative stress and peripheral muscle dysfunction in patients with chronic obstructive pulmonary disease. Am J Respir Crit Care Med 2003;167:1664-9.

4 Reid MB. Nitric oxide, reactive oxygen species, and skeletal muscle contraction. Med Sci Sports Exerc 2001;33:371-6.

5 American Thoracic Society and European Respiratory Society. Skeletal muscle dysfunction in chronic obstructive pulmonary disease. Am J Respir Crit Care Med 1999;159:S1-40.

6 Koechlin C, Couillard A, Simar D, et al. Does oxidative stress alter quadriceps endurance in chronic obstructive pulmonary disease? Am J Respir Crit Care Med 2004; 169:1022-7.

7 Raguso CA, Guinot SL, Janssens JP, et al. Chronic hypoxia: common traits between chronic obstructive pulmonary disease and altitude. Curr Opin Clin Nutr Metab Care 2004;7:411-7.

8 Hoppeler $\mathrm{H}$, Vogt $M$, Weibel ER, et al. Response of skeletal muscle mitochondria to hypoxia. Exp Physiol 2003;88:1, 109-19.

9 Chandel NS, Schumacker PT. Cellular oxygen sensing by mitochondria: old questions, new insight. J Appl Physiol 2000;88:1880-9.

10 Simon-Schnass IM. Risk of oxidative stress at high altitude and possible benefit of antioxidant supplementation. In: Sen CK, Packer L, Hanninen, eds. Handbook of oxidants and antioxidants in exercise. Amsterdam: Elsevier, 2000:555-77.

11 Bailey DM, Davies B, Young IS. Intermittent hypoxic training: implications for lipid peroxidation induced by acute normoxic exercise in active men. Clin Sci (Lond) 2001;101:465-75.

12 Martinelli M, Winterhalder R, Cerretelli P, et al. Muscle lipofuscin content and satellite cell volume is increased after high altitude exposure in humans. Experientia 1990;46:672-6.

13 Lundby $\mathrm{C}$, Pilegaard $\mathrm{H}$, van $\mathrm{Hall} \mathrm{G}$, et al. Oxidative DNA damage and repair in skeletal muscle of humans exposed to high-altitude hypoxia. Toxicology 2003; 192:229-36.

14 Serres I, Gautier V, Varray A, et al. Impaired skeletal muscle endurance related to physical inactivity and altered lung function in COPD patients. Chest 1998;113:900-5

15 Reid MB, Li YP. Cytokines and oxidative signalling in skeletal muscle. Acta Physiol Scand 2001;171:225-32.

16 Koong AC, Chen EY, Giaccia AJ. Hypoxia causes the activation of nuclear factor kappa B through the phosphorylation of I kappa B alpha on tyrosine residues. Cancer Res 1994:54:1425-30.

17 Matsui $H$, Ihara $Y$, Fujio $Y$, et al. Induction of interleukin (IL)-6 by hypoxia is mediated by nuclear factor (NF)-kappa B and NF-IL6 in cardiac myocytes. Cardiovasc Res 1999;42:104-12.

18 Gan WQ, Man SF, Senthilselvan A, et al. Association between chronic obstructive pulmonary disease and systemic inflammation: a systematic review and a meta-analysis. Thorax 2004;59:574-90.

19 Takabatake N, Nakamura H, Abe S, et al. The relationship between chronic hypoxemia and activation of the tumor necrosis factor- $\alpha$ system in patients with chronic obstructive pulmonary disease. Am J Respir Crit Care Med 2000;161:1179-84.

20 Andersen. P, Adams RP, Sjogaard G, et al. Dynamic knee extension as model for study of isolated exercising muscle in humans. J Appl Physiol 1985;59:1647-53.

21 Fabbri LM, Hurd SS. Global strategy for the diagnosis, management and prevention of COPD: 2003 update. Eur Respir J 2003;22:1-2.

22 Mellemgaard K. The alveolar arterial oxygen difference: its size and components in normal man. Acta Physiol Scand 1966;67:10-20.

23 Knudson RJ, Slatin RC, Lebowitz MD, et al. The maximal expiratory flowvolume curve: normal standards, variability and effects of age. Am Rev Respir Dis 1976;113:587-600.

24 Marquis K, Debigaré R, Lacasse Y, et al. Midthigh muscle cross-sectional area is a better predictor of mortality than body mass index in patients with chronic obstructive pulmonary disease. Am J Respir Crit Care Med 2002; 166:809-13

25 Bergström J. Electrolytes in man: determination by neutron activation analysis on needle biopsy specimens: a study on normal subjects, kidney patients and patients with chronic diarrhoea. Scand J Clin Lab Invest 1962:14:1-10.

26 Whittom F, Jobin J, Simard MA, et al. Histochemical and morphological characteristics of the vastus lateralis muscle in patients with chronic obstructive pulmonary disease. Med Sci Sports Exerc 1998;30:1467-74.

27 Mabuchi K, Sréter FA. Actomyosin ATPase: quantitative measurement of activity in cryostat sections. Muscle Nerve 1980;3:227-32.

28 Allaire J, Maltais F, LeBlanc $\mathrm{P}$, et al. Lipofuscin accumulation in the vastus lateralis muscle in patients with chronic obstructive pulmonary disease. Muscle Nerve 2002;25:383-9.

29 Ohkawa H, Ohishi N, Yagi K. Assay for lipid peroxides in animal tissues by thiobarbituric acid reaction. Anal Biochem 1979;95:351-8.

30 Nakamura W, Hosoda S, Hayashi K. Purification and properties of rat liver glutathione peroxidase. Biochim Biophys Acta 1974;358:251-61.

31 Sun M, Zigman S. An improved spectrophotometric assay for superoxide dismutase based on epinephrine autoxidation. Anal Biochem 1978:90:81-9.

32 Bland JM, Altman DG. Statistical methods for assessing agreement between two methods of clinical measurements. Lancet 1986;1:307-10.

33 Noguera A, battle $S$, Miralles $C$, et al. Enhanced neutrophil response in chronic obstructive pulmonary disease. Thorax 2001;56:432-7.

34 Oudijk EJD, Lammers JWJ, Koenderman L. Systemic inflammation in chronic obstructive pulmonary disease. Eur Respir J 2003;22:S5-13.

35 Rahman I, MacNee W. Role of transcription factors in inflammatory lung disease. Thorax 1998:53:601-12.

36 Agusti A, Morla M, Sauleda J, et al. NF-kappaB activation and iNOS upregulation in skeletal muscle of patients with COPD and low body weight. Thorax 2004;59:483-7.

37 McCord JM, Roy RS, Schaffer SW. Free radicals and myocardial ischemia. The role of xanthine oxidase. Adv Myocardiol 1985;5:183-9.

38 Heunks L, Vina J, Cees L, et al. Xanthine oxidase is involved in exerciseinduced oxidative stress in chronic obstructive pulmonary disease. Am J Physiol 1999;277:R1697-700.

39 Terasawa K, Fujiwara T, Sakai A, et al. Comparison of muscle force, muscle endurance, and electromyogram activity during an expedition at high altitude. Int J Biometeorol 1996;39:111-5.

40 Esposito F, Orizio C, Parrinello G, et al. Chronic hypobaric hypoxia does not affect electro-mechanical muscle activities during sustained maximal isometric contractions. Eur J Appl Physiol 2003:90:337-43.

41 Fulco CS, Cymerman A, Muza SR, et al. Adductor pollicis muscle fatigue during acute and chronic altitude exposure and return to sea level. J Appl Physiol 1994;77: 179-83.

42 Zattara-Hartmann MC, Badier M, Guillot C, et al. Maximal force and endurance to fatigue of respiratory and skeletal muscles in chronic hypoxemic patients: the effects of oxygen breathing. Muscle Nerve 1995; 18:495-502.

43 Sohal RS, Brunk UT. Lipofuscin as an indicator of oxidative stress and aging. Adv Exp Med Biol 1989;266:17-29.

44 Haliwell B, Chirico S. Lipid peroxidation: its mechanism, measurement and significance. Am J Clin Nutr 1993;57:715-24.

45 Levine RL, Williams JA, Stadtman ER, et al. Carbonyl assays for determination of oxidatively modified proteins. Methods Enzymol 1994;233:346-57.

46 Liu J, Yeo HC, Overvik-Douki E, et al. Chronically and acutely exercised rats: biomarkers of oxidative stress and endogenous antioxidants. J Appl Physiol 2000;89:21-8

47 Alessio HM, Goldfarb AH. Lipid peroxidation and scavenger enzymes during exercise: adaptive response to training. J Appl Physiol 1988;64:1333-6.

48 Moller $\mathbf{P}$, Loft $\mathrm{S}$, Lundby $\mathrm{C}$, et al. Acute hypoxia and hypoxic exercise induce DNA strand breaks and oxidative DNA damage in humans. FASEB $J$ 2001;15:1181-6.

49 Frankiewicz-Jozko A, Faff J, Sieradzan-Gabelska B. Changes in concentrations of tissue free radical marker and serum creatine kinase during the post-exercise period in rats. Eur J Appl Physiol 1996;74:470-4.

50 Cannon. G, St Pierre. B. Cytokines in exertion-induced skeletal muscle injury. Mol Cell Biochem 1998;179:159-67. 